\title{
Surface control of flexoelectricity
}

\author{
Massimiliano Stengel ${ }^{1,2}$ \\ ${ }^{1}$ ICREA - Institució Catalana de Recerca i Estudis Avançats, 08010 Barcelona, Spain \\ ${ }^{2}$ Institut de Ciència de Materials de Barcelona (ICMAB-CSIC), Campus UAB, 08193 Bellaterra, Spain
}

(Dated: September 12, 2018)

\begin{abstract}
The polarization response of a material to a strain gradient, known as flexoelectricity, holds great promise for novel electromechanical applications. Despite considerable recent progress, however, the effect remains poorly understood. From both the fundamental and practical viewpoints, it is of crucial importance to know whether the coupling coefficients are primarily governed by the properties of the bulk material or by the details of the sample surface. Here we provide, by means of first-principles calculations, quantitative evidence supporting the latter scenario. In particular, we demonstrate that a $\mathrm{SrTiO}_{3}$ film can yield a positive or negative flexoelectric voltage depending on its surface termination. This result points to a full control of the flexoelectric effect via surface/interface engineering, opening exciting new avenues for device design.
\end{abstract}

PACS numbers: 71.15.-m,77.65.-j

Flexoelectricity is a universal property of all insulators, whereby a macroscopic electrical polarization is generated in response to an inhomogeneous mechanical strain. 1] The recent surge of interest in this phenomenon 2] has come with the realization that strain gradients can be huge at the nanoscale, and generate a large enough polarization to rival conventional piezoelectricity. [3] Long regarded as a drawback in the operation of thin-film devices (e.g. ferroelectric memories [4], or light-emitting diodes in foldable electronics [5]), strain gradients are now being increasingly recognized as a rich playground for exploring new, potentially useful, functionalities [6, 7]. Future progress towards practical applications crucially relies on identifying the microscopic mechanism that are most effective at delivering a large electrical response, and on harnessing them via specific materials-design rules. Unfortunately, such a fundamental knowledge is currently very limited.

A central (yet vastly unexplored) question concerns the role played by the sample surfaces. Both symmetry arguments [8] and quantum-mechanical theory [9, 10] predict their contribution to be comparable to that of the bulk, regardless of sample size. This fact, in principle, calls for a substantial revision of the currently established device design strategies, where only bulk electromechanical properties (such as piezoelectricity [11] and/or electrostriction) are typically taken into account. [12] In practice, however, there are currently little or no indications on the magnitude of the aforementioned surface effects, nor on their microscopic physical nature, so the necessity for their explicit inclusion in the models remains open to debate. It appears unlikely that such indications will emerge from the experiments alone, at least in the near future: as the bulk and surface contributions scale identically as a function of sample size (unlike other interface-related phenomena in oxides, e.g. the dielectric "dead" layer [13, 14]), they appear difficult or impossible to disentangle by purely electrical means. In this context, theoretical modeling can be of great help.

First-principles electronic-structure methods are, in principle, ideally suited to shed some light on the above issues, thanks to their unbiased predictive power. Indeed, building on the work of Resta [15], Hong and Vanderbilt [9, 16] have recently devised a promising route to addressing the flexoelectric problem at a fundamental quantum-mechanical level, and applied it to calculating the response properties of a number of bulk materials in the framework of density-functional theory (DFT). However, even at the bulk level, a complete determination of the full flexoelectric tensor has not been achieved yet, as the electronic contribution to the transversal components is still missing [16]. Furthermore, the impact of surface effects was not considered in refs. 9, 16], nor in any other first-principles study [17, 18] reported to date. Very recent advances promoted by the author [10, 19] have now opened the way to filling both gaps, by combining density-functional perturbation theory (DFPT) -the linear-response version of DFT- with a covariant formulation of electrostatics in the coordinate system of the deformed body. Here we use such a methodology to study the flexoelectric response of $\mathrm{SrTiO}_{3}$, arguably the most important material for applications, and the best known experimentally [20]. Our results, in addition to providing a complete physical picture of the effect, demonstrate that the surface indeed matters: by modifying the atomically thin termination layer, one can tune -and even reverse - the voltage response of a macroscopically thick film.

The flexoelectric performance of an insulating material can be conveniently quantified as the open-circuit voltage, $\Delta V$, that is linearly induced by a strain-gradient deformation [see Fig. 1(c)] in the limit of a large film thickness, $t$,

$$
\varphi_{x \lambda, \beta \gamma}=\lim _{t \rightarrow \infty} \frac{1}{t} \frac{\partial \Delta V}{\partial \varepsilon_{\beta \gamma, \lambda}}
$$


a

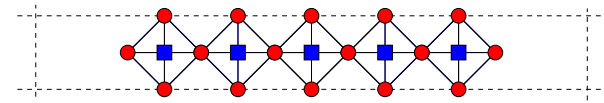

b

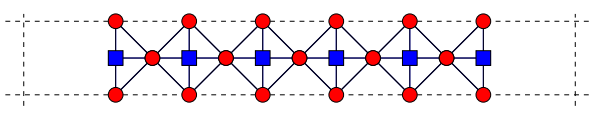

C

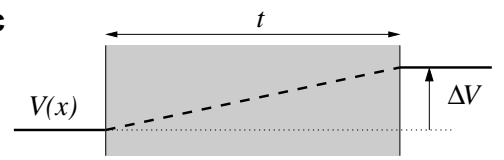

d

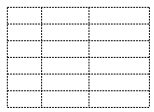

e

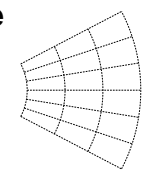

f

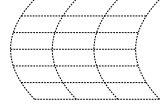

FIG. 1: Schematic illustration of the computational setup. Panels $(a, b)$ show the supercell models of the SrO- (a) and $\mathrm{TiO}_{2}$-terminated (b) $\mathrm{SrTiO}_{3}$ slabs ( $\mathrm{Ti}$ and $\mathrm{O}$ atoms are represented as squares and circles; $\mathrm{Sr}$ atoms are not shown). Panel (c) shows the induced open-circuit voltage, $\Delta V$, induced by a longitudinal $\left(\varepsilon_{x x, x}, \mathrm{~d}\right)$, transversal $\left(\varepsilon_{y y, x}, \mathrm{e}\right)$ or shear $\left(\varepsilon_{x y, y}\right.$, f) strain-gradient deformation.

Here $\varepsilon_{\beta \gamma, \lambda}=\partial \varepsilon_{\beta \gamma} / \partial r_{\lambda}$ is the gradient of the symmetric strain tensor $\left(\varepsilon_{\beta \gamma}\right)$ along the Cartesian direction $r_{\lambda}$, and $x$ indicates the direction normal to the surface; the relevant components of $\varepsilon_{\beta \gamma, \lambda}$ in the context of this work are illustrated in Fig. 1(d-f). Remarkably, although $\varphi_{x \lambda, \beta \gamma}$ (usually referred to as "flexocoupling" coefficient) is a macroscopic response property of the system, it is known [10] to contain both bulk- and surface-specific contributions,

$$
\varphi_{x \lambda, \beta \gamma}=\varphi_{x \lambda, \beta \gamma}^{\mathrm{bulk}}+\varphi_{x \lambda, \beta \gamma}^{\mathrm{surf}} .
$$

The former term is given by $\varphi_{x \lambda, \beta \gamma}^{\text {bulk }}=\mu_{x \lambda, \beta \gamma}^{\text {bulk }} /\left(\epsilon_{0} \epsilon_{x x}\right)$, where $\boldsymbol{\mu}^{\text {bulk }}$ is the bulk flexoelectric tensor, $\boldsymbol{\epsilon}$ is the macroscopic dielectric tensor and $\epsilon_{0}$ is the vacuum permittivity. The latter term, $\varphi_{x \lambda, \beta \gamma}^{\text {surf }}$, originates from surface piezoelectric effects, [8, 10] which are present in any material regardless of crystal symmetry.

While techniques for calculating both quantities in a first-principles context have recently been proposed, 10, 16, 19] the determination of the bulk flexoelectric tensor remains challenging, particularly concerning the purely electronic contributions. In fact, directly calculating the bulk polarization response to a strain gradient would require access to the microscopic current density [16, 19] induced by a deformation, whose code implementation is not available yet. To overcome this methodological obstacle (and solve for the missing transversal components of $\varphi^{\text {bulk }}$ ) we shall compute, rather than the polarization response of the bulk, the internal electric field response of a slab. The advantage of the latter approach is that it can be carried out with the sole knowledge of the firstorder charge density. [19] For the sake of computational convenience, we shall initially focus on "frozen-ion" (in

the sense specified in ref. [10]) deformation of "truncatedbulk" slabs, i.e., with the unperturbed atoms placed at their ideal lattice sites. The impact of full ionic relaxation, which is essential for a quantitative analysis of the flexoelectric effect, is uncomplicated to calculate once the electronic contributions to the bulk tensor are known, and will be dealt with in a later part of this work.

We consider the supercell models illustrated in Fig. 1(a-b) (i.e. periodically repeated sequences of symmetrically terminated $\mathrm{SrTiO}_{3}$ slabs and vacuum layers), and proceed as follows. First, we calculate how the microscopic charge density of the supercell, $\rho(\mathbf{r})$, responds to a selected set of long-wavelength acoustic phonons, by using DFPT as implemented in the ABINIT [21, 22. package. Next, we perform a Taylor expansion (in the wavevector $\mathbf{q}$ ) of such density response functions, along the lines described in refs. [10, 19]. This analysis readily yields the response to a macroscopic strain gradient in the curvilinear coordinate system of the deformed crystal lattice; 10] in particular, one has

$$
\frac{\partial \rho(\mathbf{r})}{\partial \varepsilon_{\beta \gamma, \lambda}}=r_{\lambda} \rho_{\beta \gamma}^{\mathrm{U}}(\mathbf{r})+\rho_{\lambda, \beta \gamma}^{\mathrm{G}}(\mathbf{r}),
$$

where $\rho^{\mathrm{U}, \mathrm{G}}$ are cell-periodic functions $\left[\rho^{\mathrm{U}}=\partial \rho(\mathbf{r}) / \partial \varepsilon_{\beta \gamma}\right.$ describes the response to a uniform strain, while $\rho^{\mathrm{G}}$ is the additional contribution that is due to the gradient]. Finally, we use $\rho^{\mathrm{U}}$ and $\rho^{\mathrm{G}}$ to calculate the induced electric field. In the curvilinear frame, the first-order $\mathbf{E}$ is related to the first-order $\rho$ via the modified [10] Gauss's law,

$$
\nabla \cdot\left(\frac{\partial \mathbf{E}(\mathbf{r})}{\partial \varepsilon_{\beta \gamma, \lambda}}+r_{\lambda} \mathbf{E}_{\beta \gamma}^{\mathrm{met}}(\mathbf{r})\right)=\frac{1}{\epsilon_{0}} \frac{\partial \rho(\mathbf{r})}{\partial \varepsilon_{\beta \gamma, \lambda}}
$$

where the metric contribution $\mathbf{E}^{\text {met }}$ depends on the unperturbed electric field $\mathbf{E}$ as

$$
E_{\alpha, \beta \gamma}^{\mathrm{met}}=\delta_{\beta \gamma} E_{\alpha}-\delta_{\gamma \alpha} E_{\beta}-\delta_{\alpha \beta} E_{\gamma} .
$$

As the electric field is related to the potential by $\mathbf{E}(\mathbf{r})=$ $-\nabla V(\mathbf{r})$, knowledge of the former (and of its first-order variation, $\left.\partial \mathbf{E}(\mathbf{r}) / \partial \varepsilon_{\beta \gamma, \lambda}\right)$ then yields the linear variation of the latter, $\partial V(\mathbf{r}) / \partial \varepsilon_{\beta \gamma, \lambda}$, and ultimately the soughtafter values of the flexocoupling coefficients, $\varphi$.

In practice, to solve Eq. (4), it is convenient to work with the "macroscopic averages" 23, 24] of the $\mathbf{E}$ and $\rho$ response functions, where the oscillations that occur on the scale of the interatomic spacings have been appropriately filtered out. This procedure has two advantages: first, it allows one to identify the relevant electrical properties of the system in a macroscopic context (e.g. internal fields, surface potential offsets, etc.); second, it facilitates the implementation of the Poisson solver by making the problem one-dimensional. In particular, in close analogy to Eq. (3), one can write the normal $(x)$ 

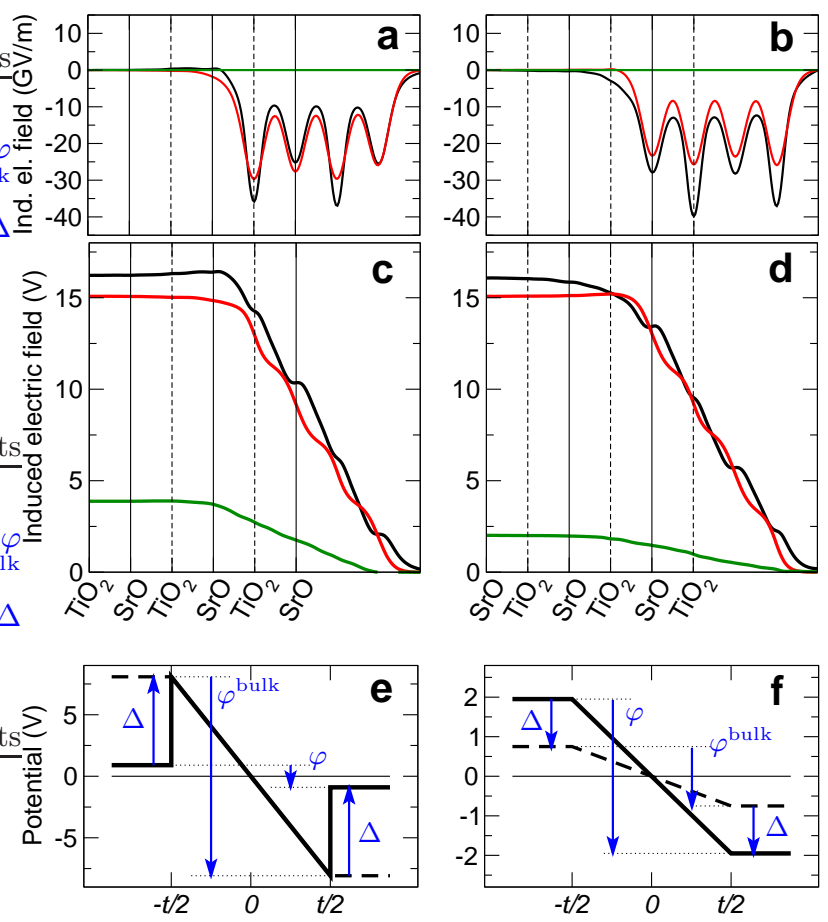

FIG. 2: Electric field and potential response to mechanical deformations. The $E_{x}^{\mathrm{U}}(\mathrm{a}-\mathrm{b})$ and $E_{x}^{\mathrm{G}}(\mathrm{c}-\mathrm{d})$ response functions are shown for a $\mathrm{SrO}-(\mathrm{a}, \mathrm{c})$ and $\mathrm{TiO}_{2}$-terminated (b,d) slab. Black, red and green curves refer to longitudinal, transversal and shear deformations, respectively. The location of the $\mathrm{SrO}$ (dashed) and $\mathrm{TiO}_{2}$ (solid) atomic layers is indicated by vertical lines (only half of the symmetric slab is shown). Panels (e-f) show the electrostatic potential that would be induced in a macroscopic SrO-terminated slab of thickness $t$ when subjected to a strain gradient of hypotetical magnitude $1 / t$. The longitudinal (e) and shear (f) cases are shown, illustrating the qualitative difference in the response. Dashed and solid lines refer to the bulk and total contribution, respectively; $\Delta=\varphi^{\text {surf }} / 2$.

component of the macroscopically averaged E-field response as

$$
\begin{aligned}
& \frac{\partial E_{x}(x)}{\partial \varepsilon_{\beta \beta, x}}=x E_{x, \beta \beta}^{\mathrm{U}}(x)+E_{x x, \beta \beta}^{\mathrm{G}}(x), \\
& \frac{\partial E_{x}(x)}{\partial \varepsilon_{x y, y}}=E_{x y, x y}^{\mathrm{G}}(x),
\end{aligned}
$$

where Eq. (6) refers to either the longitudinal or transversal case $(\beta=x, y)$, and Eq. (77) concerns a shear deformation. Note that the parallel components of the induced E-field vanish, hence the exclusive focus on $E_{x}$. Note also the absence of the uniform-strain contribution in Eq. (7): $E_{x, x y}^{\mathrm{U}}(x)$ vanishes identically in a centrosymmetric slab.

In Fig. 2(a-d) we plot the calculated $E_{x}^{\mathrm{U}, \mathrm{G}}(x)$, corresponding to either a $\mathrm{SrO}$ - or a $\mathrm{TiO}_{2}$-terminated slab and to each of the three types of strain gradients shown in Fig. 1(d-f). (We refer the reader to Supplementary Note 1 for the details of the computational methodology and parameters.) As anticipated in Eqs. (6) and (7), there is an important qualitative difference between the longitudinal or transversal response, where the strain gradient is oriented along the surface normal, and the shear response, where it is directed in plane.

In the former two cases, $E_{x, \beta \beta}^{\mathrm{U}}(x)$ is roughly uniform and negative (the oscillations are irrelevant on a macroscopic scale) in a thin region surrounding the surface layer. This is consistent with the expected behavior of the electrostatic potential upon uniform deformation of the slab: the unperturbed $V(x)$ is a symmetric potential well, whose depth, $\phi$, is modified by a diagonal strain component, $\varepsilon_{\beta \beta}$. Such a dependence of $\phi$ on the strain corresponds precisely to the surface contribution to the flexocoupling coefficient,

$$
\varphi_{x x, \beta \beta}^{\text {surf }}=\frac{d \phi}{d \epsilon_{\beta \beta}}=-\int_{0}^{+\infty} d x E_{x, \beta \beta}^{\mathrm{U}}(x) .
$$

The functions $E_{x x, \beta \beta}^{\mathrm{G}}(x)$, describing the genuine straingradient effects, display a capacitor-like behavior: the field is uniform inside the film and zero outside, consistent with the open-circuit electrical boundary conditions (EBC) that were enforced. Interestingly, the internal field, $E_{x x, \beta \beta}^{\mathrm{slab}}=E_{x x, \beta \beta}^{\mathrm{G}}(x=0)$, appears to be independent of the surface termination. This is not a coincidence: the open-circuit flexoelectric field in the longitudinal and transversal case is a bulk property of the material, and relates to the bulk flexocoupling coefficients [10, 19] as

$$
\varphi_{x x, \beta \beta}^{\text {bulk }}=-E_{x x, \beta \beta}^{\mathrm{slab}} .
$$

Thus, for a strain gradient of the type $\varepsilon_{\beta \beta, x}$, the analysis of the E-response of the deformed slab yields complete information on both surface and bulk contributions to the flexoelectric effect [their respective impact on the electrostatic potential of a macroscopic film is illustrated in Fig. 2(e)]. Most importantly, we have thereby gained access to the transversal component of the bulk flexocoupling coefficient, $\varphi_{x x, y y}^{\text {bulk }}$, whose computation has eluded earlier first-principles attempts.

In the shear case, the flexoelectric field depends on both bulk and surface-specific properties, [10] and is therefore termination-dependent [see Fig. 2(f)]; from the electric field response functions of Fig. 22(a-d) we can thus only extract the total flexocoupling coefficient of the slab, $\varphi_{x y, x y}=-E_{x y, x y}^{\text {slab }}$. To separate $\varphi_{x y, x y}$ into bulk and surface terms it suffices, however, to complement the above data with a calculation of bulk $\mathrm{SrTiO}_{3}$. (Details are reported in Supplementary Note 2.) The latter, in particular, yields two additional response quantities, [16]

$$
\begin{aligned}
& \varphi_{\mathrm{L} 1}^{\text {bulk }}=\varphi_{x x, x x}^{\text {bulk }}, \\
& \varphi_{\mathrm{L} 2}^{\text {bulk }}=\varphi_{x x, y y}^{\text {bulk }}+2 \varphi_{x y, x y}^{\text {bulk }} .
\end{aligned}
$$

Eq. (10) constitutes a useful consistency check of the methodology, as $\varphi_{\mathrm{L} 1}^{\text {bulk }}$ is redundant with the already calculated value of $\varphi_{x x, x x}^{\text {bulk }}$. Eq. (11), on the other hand, 


\begin{tabular}{lrrrrr}
\hline \hline & \multicolumn{2}{c}{$\varphi^{\text {bulk }}$} & \multicolumn{2}{c}{$\varphi^{\text {surf }}$} & \multicolumn{2}{c}{$\varphi($ total) } \\
& & \multicolumn{1}{c}{$\mathrm{SrO}$} & $\mathrm{TiO}_{2}$ & \multicolumn{1}{c}{$\mathrm{SrO}$} & \multicolumn{1}{c}{$\mathrm{TiO}_{2}$} \\
\hline$x x, x x(\mathrm{~L})$ & -16.153 & 14.356 & 16.948 & -1.797 & 0.795 \\
$x x, y y(\mathrm{~T})$ & -15.075 & 15.683 & 12.447 & 0.608 & -2.628 \\
$x y, x y(\mathrm{~S})$ & -1.495 & -2.382 & -0.515 & -3.877 & -2.010 \\
\hline \hline
\end{tabular}

TABLE I: Frozen-ion flexocoupling coefficients of a truncatedbulk $\mathrm{SrTiO}_{3}$ slab. To compute $\varphi^{\text {bulk }}$ we combined the two quantities that we extracted from the bulk calculations, $\varphi_{\mathrm{L} 1}^{\mathrm{bulk}}=-16.15 \mathrm{~V}$ and $\varphi_{\mathrm{L} 2}^{\mathrm{bulk}}=-18.07 \mathrm{~V}$ (these are in excellent agreement with the values reported by Hong and Vanderbilt [16]: $\varphi_{\mathrm{L} 1}^{\text {bulk }}=-16.25 \mathrm{~V}$ and $\left.\varphi_{\mathrm{L} 2}^{\text {bulk }}=-18.17 \mathrm{~V}\right)$, with the value of $E_{x x, y y}^{\text {slab }}=15.08 \mathrm{~V}$, which we extracted from Fig. 2(cd). The other values have been obtained as explained in the text. (L), (T) and (S) stands for longitudinal, transversal and shear, respectively. Volt units are used throughout.

yields the sought-after value of $\varphi_{x y, x y}^{\text {bulk }}$ since we already know $\varphi_{x x, y y}^{\text {bulk }}$ from the slab calculations. Finally, we use $\varphi_{x y, x y}=-E_{x y, x y}^{\text {slab }}$ to infer $\varphi_{x y, x y}^{\text {surf }}=\varphi_{x y, x y}-\varphi_{x y, x y}^{\text {bulk }}$.

Our results for the bulk, surface, and total flexocoupling coefficients of the truncated-bulk, frozen-ion deformation of a $\mathrm{SrTiO}_{3}$ slab are summarized in Table I At the bulk level, it is interesting to note the relatively small magnitude of the shear coefficients, $\varphi_{x y, x y}^{\text {bulk }}$ and $\varphi_{x y, x y}^{\text {surf }}$, compared to both the longitudinal and the transversal ones. Meanwhile, in the latter two cases there is a large cancellation between bulk and surface terms; as a result, the values of the total flexocoupling coefficients, $\varphi$, are all comparable in magnitude. This fact can be rationalized by observing that the linear response to atomic displacements, in a ionic (or partially ionic) solid, is largely dominated by the rigid displacement of an approximately spherical charge density distribution surrounding each atom. The spherical contribution, which is typically large and negative, 9] shows up in $\varphi_{x x, \beta \beta}^{\text {bulk }}$, and with opposite sign in $\varphi_{x x, \beta \beta}^{\text {surf }}$ in the shear case neither the bulk nor the surface term are affected. (See Supplementary Note 1 of Ref. 10 and Figure S2 therein.) Remarkably, the resulting values of $\varphi$ depend strongly on the details of the surface, and in some cases even have opposite signs in the $\mathrm{SrO}$ - and $\mathrm{TiO}_{2}$-terminated slabs. Such a conclusion, in fact, persists after we take into account the full relaxation of the atomic structure; we shall prove this point in the following paragraphs.

To investigate the relaxed-ion response of the film, we shall consider an "effective" bending deformation of the type [10]

$$
\varepsilon_{\mathrm{eff}, x}=\varepsilon_{y y, x}-\nu \varepsilon_{x x, x}
$$

The coefficient $\nu=\mathcal{C}_{x x, y y} / \mathcal{C}_{x x, y y}$ is the ratio of the transversal and longitudinal components of the bulk elastic tensor, $\mathcal{C}_{\alpha \beta \gamma \lambda}$, and accounts for the mechanical equilibrium condition, i.e. it ensures that the stress field van-

\begin{tabular}{|c|c|c|c|c|c|}
\hline & \multirow[t]{2}{*}{$\varphi^{\text {bulk }}$} & \multicolumn{2}{|c|}{$\varphi^{\text {surf }}$} & \multicolumn{2}{|c|}{$\varphi($ total $)$} \\
\hline & & $\mathrm{SrO}$ & $\mathrm{TiO}_{2}$ & $\mathrm{SrO}$ & $\mathrm{TiO}_{2}$ \\
\hline $\mathrm{FI}$ & -10.368 & 13.468 & 6.837 & 3.100 & -3.531 \\
\hline LM & -0.444 & -4.934 & 5.343 & -5.378 & 4.899 \\
\hline $\mathrm{RI}$ & -10.812 & 8.534 & 12.180 & -2.278 & 1.368 \\
\hline
\end{tabular}

TABLE II: Flexocoupling coefficients of a relaxed $\mathrm{SrTiO}_{3}$ slab. The frozen-ion (FI), lattice-mediated (LM) and total relaxed-ion $(\mathrm{RI}=\mathrm{FI}+\mathrm{LM})$ values of the bulk, surface and total slab response are reported. $\varphi^{\text {bulk }}(\mathrm{FI})$ was inferred by using the $\varphi_{x x, \beta \beta}^{\text {bulk }}$ values of Table [ and the calculated $\nu$ parameter. The $\varphi_{x x, \text { eff }}^{\text {surf }}(\mathrm{FI})$ values, however, do not correspond to the linear combination of $\varphi_{x x, x x}^{\text {surf }}$ and $\varphi_{x x, y y}^{\text {surf }}$ from Table $\llbracket$ the $\varphi_{x x, \text { eff }}^{\text {surf }}(\mathrm{FI})$ reported here refer to the frozen-ion deformation of a relaxed, rather than truncated-bulk, surface geometry. Volt units are used throughout.

ishes everywhere in the interior of the deformed sample. (Our calculated value for $\mathrm{SrTiO}_{3}$ is $\nu=0.2914$.) As before, the overall flexocoupling coefficient of the slab can be written in terms of a bulk and a surface contribution,

$$
\varphi_{x x, \mathrm{eff}}=\varphi_{x x, \mathrm{eff}}^{\mathrm{bulk}}+\varphi_{x x, \mathrm{eff}}^{\mathrm{surf}},
$$

whose respective impact on the induced electrostatic potential follow the form of Fig. 2(e). The relaxed-ion (RI) value of $\varphi_{x x, \mathrm{eff}}^{\text {bulk }}$ consists in a frozen-ion (FI) contribution, given in terms of the already known $\varphi_{x x, \beta \beta}^{\text {bulk }}$ values, plus a lattice-mediated (LM) part, which we calculate as detailed in Supplementary Note 2. The corresponding contributions to $\varphi_{x x \text {,eff }}^{\text {surf }}$ are calculated by applying a uniform strain of the type $\varepsilon_{\text {eff }}=\varepsilon_{y y}-\nu \varepsilon_{x x}$ to fully relaxed slab supercells (see Supplementary Note 3).

A summary of the results is reported in Table I. The values marked with boldface font, i.e., the flexocoupling coefficient of a fully relaxed $\mathrm{SrTiO}_{3}$ slab subjected to bending, are the main result of this work. [The beambending case is easily recovered by multiplying the reported values by $\tau=\mathcal{C}_{x x, x x} /\left(\mathcal{C}_{x x, x x}+\mathcal{C}_{x x, y y}\right)$. By using the calculated elastic constants of bulk $\mathrm{SrTiO}_{3}$, reported in Table S2, we find $\tau=0.77$.] Note their substantial departure with respect to the corresponding bulk coefficient, confirming the dramatic impact of the surface structural and electronic properties on the electromechanical response of the system. Remarkably, the aforementioned response coefficients are opposite in sign depending on whether a $\mathrm{SrO}$ - and $\mathrm{TiO}_{2}$-terminated slab is considered. In fact, the surface shows an even larger termination dependence at the frozen-ion level, but with opposite sign: the LM contribution to $\varphi^{\text {surf }}$ depends so strongly on the termination that its inclusion results in a voltage reversal, both in the $\mathrm{TiO}_{2}$ - and SrO-type slabs. (A microscopic analysis of the surface relaxations is provided in Supplementary Note 3.) By contrast, the LM contribution to the bulk flexocoupling coefficient is rela- 
tively minor, about one order of magnitude smaller than any other value reported in the table, and of little impact on the final results. This constitutes a substantial departure from the commonly accepted idea that bulk lattice-mediated mechanisms are predominantly responsible for the flexoelectric polarization. On the contrary, our results indicate that, by modifying the surface, one can fully control the magnitude, and even the sign, of the flexoelectric effect, in stark contrast with previous assumptions.

These results have profound implications, both for the interpretation of the experiments and for the optimization of electromechanical devices based on the flexoelectric effect. In particular, achieving a control over the properties of the sample surface appears crucial to maximizing the flexoelectric performance of a material like $\mathrm{SrTiO}_{3}$. Given the rich variety of surface structures and compositions that are accessible to perovskite oxides depending on thermodynamic conditions and treatment procedures, this opens up an essentially unlimited range of opportunities for device design.

Acknowledgments. We thankfully acknowledge the computer resources, technical expertise and assistance provided by the Supercomputing Center of Galicia (CESGA).

[1] A. K. Tagantsev, Phys. Rev. B 34, 5883 (1986).

[2] P. Zubko, G. Catalan, and A. K. Tagantsev, Annu. Rev. Mater. Res. 43, 387 (2013).

[3] L. E. Cross, J. Mater. Sci. 41, 53 (2006).

[4] G. Catalan, B. Noheda, J. McAneney, L. J. Sinnamon, and J. M. Gregg, Phys. Rev. B 72, 020102 (2005).

[5] S.-I. Park, A.-P. Le, J. Wu, Y. Huang, X. Li, and J. A.
Rogers, Advanced Materials 22, 3062 (2010).

[6] G. Catalan, A. Lubk, A. H. G. Vlooswijk, E. Snoeck, C. Magen, A. Janssens, G. Rispens, G. Rijnders, D. H. A. Blank, and B. Noheda, Nature Materials 10, 963 (2011).

[7] H. Lu, C.-W. Bark, D. E. de los Ojos, J. Alcala, C. B. Eom, G. Catalan, and A. Gruverman, Science 336, 59 (2012).

[8] A. K. Tagantsev and A. S. Yurkov, Journal of Applied Physics 112, 044103 (pages 7) (2012).

[9] J. Hong and D. Vanderbilt, Phys. Rev. B 84, 180101(R) (2011).

[10] M. Stengel, Nature Communications 4, 2693 (2013).

[11] R. M. Martin, Phys. Rev. B 5, 1607 (1972).

[12] L. C. Lew Yan Voon and M. Willatzen, J. Appl. Phys. 109, 031101 (2011).

[13] M. Stengel and N. A. Spaldin, Nature (London) 443, 679 (2006).

[14] M. Stengel, D. Vanderbilt, and N. A. Spaldin, Nature Materials 8, 392 (2009).

[15] R. Resta, Phys. Rev. Lett. 105, 127601 (2010).

[16] J. Hong and D. Vanderbilt, Phys. Rev. B 88, 174107 (2013).

[17] R. Maranganti and P. Sharma, Phys. Rev. B 80, 054109 (2009).

[18] J. Hong, G. Catalan, J. F. Scott, and E. Artacho, J. Phys.: Condens. Matter 22, 112201 (2010).

[19] M. Stengel, Phys. Rev. B 88, 174106 (2013).

[20] P. Zubko, G. Catalan, A. Buckley, P. R. L. Welche, and J. F. Scott, Phys. Rev. Lett. 99, 167601 (2007).

[21] X. Gonze, B. Amadon, P.-M. Anglade, J.-M. Beuken, F. Bottin, P. Boulanger, F. Bruneval, D. Caliste, R. Caracas, M. Côté, et al., Computer Phys. Commun. 180, 2582 (2009).

[22] X. Gonze, Phys. Rev. B 55, 10337 (1997).

[23] A. Baldereschi, S. Baroni, and R. Resta, Phys. Rev. Lett. 61, 734 (1988).

[24] J. Junquera, M. H. Cohen, and K. M. Rabe, J. Phys.: Condens. Matter 19, 213203 (2007). 